\title{
G. I. Barenblatt in Memoriam (1927-2018)
}

Michiel Bertsch (University of Rome Tor Vergata, Italy), Alexandre J. Chorin (University of California at Berkeley, USA), Nigel Goldenfeld (University of Illinois at Urbana-Champaign, USA) and Juan Luis Vázquez (Universidad Autónoma de Madrid, Spain)

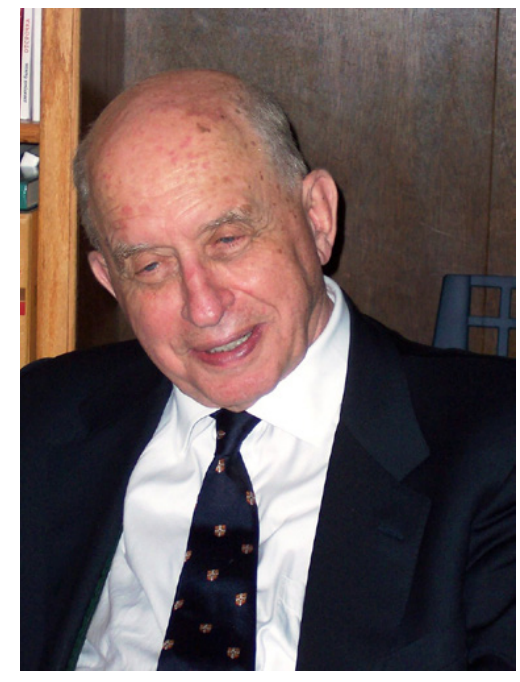

Grigory Isaakovich Barenblatt, December 2005.
The great mathematician and physicist Grigory Isaakovich Barenblatt died in Moscow on 22 June at almost 91 years of age, in the city where he was born on 10 July 1927. We say goodbye with great sorrow to the Master who taught us the beauty of selfsimilarity applied to flows and fractures and to whom some of us have dedicated so many mathematical solutions that bear his name.

Barenblatt (Grisha to his friends) was the son of the virologist Nadezhda Veniaminovna Kagan, who developed a vaccine against encephalitis and who became infected and died in a laboratory accident, and the Moscow endocrinologist Isaak Grigorievich Barenblatt. His grandfather was the mathematician Veniamin Kagan. He was the son-in-law of Pelagueya Yakovlevna Polubarinova-Kochina, illustrious pioneer of Soviet applied mathematics, who reached her centenary without stopping her work. His younger brother on his mother's side is the well-known mathematician Yakov Grigorievich Sinai, who received the Abel Prize in 2014. These data introduce us to the great Moscovite intelligentsia of the last century and they also speak to us of women with a strong imprint on science.

\section{The early years}

Grigory Isaakovich graduated in 1950 from Lomonosov Moscow State University, in the famous Department of Mechanics and Mathematics where he studied under Boris Moiseevich Levitan and Andrei Nikolaevich Kolmogorov. He obtained his first doctorate degree (Kandidat Nauk) in 1953 from that university under the supervision of Kolmogorov, one of the greatest mathematicians of the 20th century and someone who had a deep influence on him. Kolmogorov and his collaborators created the cornerstone of all turbulence research - the Kolmogorov-Obukhov analysis of the inertial range of scales, the intermediate range between the energy-containing scales and the scales where dissipation occurs - and also produced the first practical mathematical models of turbulent flows of engineering interest. Barenblatt maintained a lifelong passionate interest in these topics, as well as in dimensional analysis and scaling, tools Kolmogorov used in his analyses and whose offshoots have acquired an enormous role in contemporary mathematical physics. He was also greatly influenced by a long collaboration with Yakov Borisovich Zel'dovich, which later led to a seminal generalisation of the approach that Kolmogorov had used, uncovering the relationship between similarity and asymptotics. This work would be the cornerstone of many contributions to continuum mechanics, including fluid flow in porous media or elasto-plastic porous media, turbulence and fracture.

Grigory Isaakovich received the title of Doktor Nauk (second doctorate, a post-doctoral degree equivalent to a British DSc or a French habilitation à diriger des recherches) in 1957. In 1962, he became a professor, also at Moscow State University.

He has held the following positions in the USSR: 
1953-61 Research Scientist, Senior Research Scientist, Institute of Petroleum, USSR Academy of Sciences.

1961-75 Head, Department of Plasticity, Institute of Mechanics, Moscow University.

1975-92 Head, Theoretical Department, Institute of Oceanology, USSR Academy of Sciences.

Barenblatt's main contributions to science in the Soviet Union are noted below.

\section{Fracture mechanics:}

- Fundamental mathematical model of elastic body with cracks based on the explicit introduction of cohesion forces and solutions of basic problems.

- Introduction of one of the basic characteristics of fracture toughness: cohesion modulus.

- Basic model of the kinetics of crack propagation.

- Applications to fracture problems in metals, rocks and polymers.

- Similarity laws for brittle and quasi-brittle fracture.

- Scaling laws for fatigue cracks and multiple fractures and model of small fatigue cracks.

- Mathematical model of non-local damage accumulation.

- Mathematical model of self-oscillation and self-similar phenomena in fatigue fracture.

Theory of fluid and gas flows in porous media:

- Fundamental model of flow in fissurised porous rocks and solutions of basic problems.

- Asymptotic solutions of basic problems of unsteady groundwater and gas flow in porous media.

- Fundamental model of fluid flow in elasto-plastic porous media and solutions of basic problems.

- Non-equilibrium two-phase flow in porous media (capillary imbibition, water-oil displacement and solid phase precipitation): basic mathematical model and fundamental solutions.

- Mathematical model of gas-condensate flow in fissurised porous media.

- Mathematical model of very intense pulse in groundwater flow in porous and fissurised porous rocks.

Mechanics of non-classical deformable solids:

- Mathematical models of neck propagation in polymers (with an analogue to flame propagation) and of thermal vibro-creep in polymers.

- Mathematical model of the impact of a viscoplastic body on a rigid obstacle.

\section{Turbulence:}

- Turbulence in stratified fluids.

- Mathematical models of the transport of heavy particles in turbulent flow.

- Basic model of turbulent patch dynamics in stably stratified fluids and self-similar asymptotic laws; relations to oceanic microstructure.

- Mathematical model of non-steady heat and mass transfer in stably stratified turbulent flows.
- Model of turbulent drag reduction by polymeric additives.

- Mathematical models of turbulent bursts and turbulent shearless wake evolution.

- Mathematical model of laminar-turbulent transition taking into account the evolution of pre-existing turbulence.

- Mathematical model of temperature step formation in stably stratified turbulent flows.

- Scaling laws for developed turbulent shear flows, in particular for pipe and boundary-layer flows and walljets.

- Mathematical models of dust storms and tropical hurricanes.

\section{Self-similarity:}

- Nonlinear waves and intermediate asymptotics (longterm work generally performed in close collaboration with Ya.B. Zel'dovich).

- Concepts of intermediate asymptotics and self-similar asymptotics of the first and second kinds.

- Nonlinear eigenvalue problems.

- Relations between intermediate asymptotics and renormalisation groups.

- Basic model of the stability of self-similar solutions and travelling waves.

- Contributions to the theory of combustion and thermal explosion.

- New model of surface-tension-driven thin films.

\section{Barenblatt abroad}

With the glasnost movement, the doors were opened in the USSR and Grigory Isaakovich arrived in the West in 1990, visiting the Université de Paris VI. In 1991, he spent the Spring at the IMA Institute of the University of Minnesota, where two of the authors of this article (MB, JLV) first met him. He also met an old friend, Shoshana Kamin, a professor in Tel Aviv, who received her mathematical education in Oleinik's PDE group in Moscow. It was a stellar moment in which Barenblatt presented his ideas and posed multiple mathematical problems that would occupy researchers in our countries for years. He came from the "World on the Other Side", with his applicable equations, a permanent smile and an endless flow of stories. He sought to unite in science the best of both worlds and his life is an example that shows that it is possible. A number of the attendees reoriented their research to welcome his ideas and mathematical problems and have been solving some of those problems and raising new ones ever since. Since that Spring in Minnesota in 1991, they never lost scientific and human contact.

Then, Barenblatt became G.I. Taylor Professor of Fluid Mechanics at the University of Cambridge from 1992 to 1994 and has been G.I. Taylor Professor Emeritus of Fluid Mechanics since then. He held this to be his highest honour and the stay in Cambridge affected him deeply. Nobody understood better than he the importance of the longstanding British tradition in fluid mechanics and, with several of its representatives, such 
as Batchelor, Crighton and Lighthill, he had a deep scientific and human relationship. As an active Professorial Fellow of the Gonville \& Caius College in Cambridge, he enjoyed British academic traditions in general. He was a visiting professor at the University of Rome Tor Vergata (1992) and at the University of Minnesota (1994). In 1993, he visited the Department of Mathematics of the Universidad Autónoma of Madrid as a BBVA Visiting Professor. He returned to Spain in 1996 as Iberdrola Visiting Professor at the Universidad Autónoma de Madrid.

Grigory Isaakovich arrived in Berkeley in February 1996 as a visiting professor, after a stay at the University of Illinois. This was when another of the article's authors (AJC) met him; this was the beginning of a long collaboration and friendship.

It soon became clear that the Mathematics Department at Berkeley would be delighted to have Grigory Isaakovich for a much longer stay as Professor in Residence, a highly honoured position that does not require a heavy teaching load, with a concurrent appointment as a mathematician at the Lawrence Berkeley National Laboratory. In particular, many of the applied mathematicians at Berkeley were oriented towards computing and were enthusiastic to collaborate with a great master who had a different perspective. When he came to Berkeley, Grigory Isaakovich was already committed to a semester-long visit to Stanford, so he went there for a few months and then returned to Berkeley.

The mathematics group at the Berkeley Lab had a suite of offices where the doors were open and it was easy for faculty, postdocs and students to talk and collaborate. Grigory Isaakovich fit wonderfully into this environment. He provided advice, information, perspective and leadership. He used to invite the young mathematicians to afternoon tea in his office where he talked to them about great scientific problems, about his career and life and about science in the Soviet Union. They loved it, stayed for hours and asked questions; he gave them a perspective on the joys and possibilities of a great scientific career and a model to emulate. He taught some extraordinary courses, on topics such as fluid mechanics, fracture, turbulence and porous media; a large part of his audience was made up of faculty, from a variety of departments. He became an essential part of the applied mathematics seminar; some adaptation was needed because traditionally scientific seminars in Russia tend to be more confrontational than the ones in the United States and Grigory Isaakovich could be quite critical of poor presentations. Under his impact, the seminars became more lively, more interesting and more instructive.

Grigory Isaakovich excelled at linking different worlds, such as Russian and Western science. His major pedagogical impact at Berkeley was to link computing people to asymptotics and scaling. This produced better scientists and its impact is growing.

Grigory Isaakovich returned to live full-time with his family in Moscow in his last years. At 90, he still went to work in his Oceanology Laboratory every day he could.

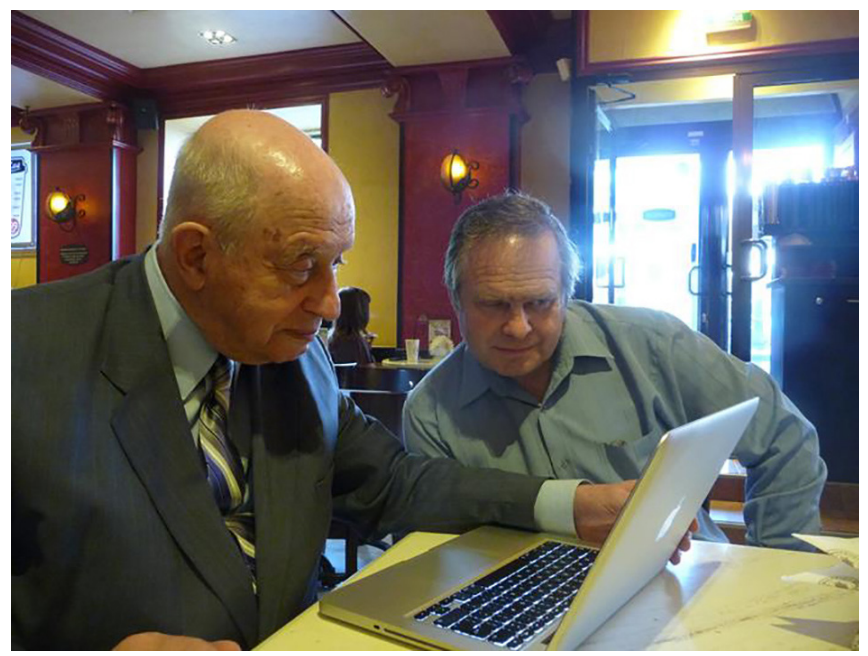

With his collaborator Prostokishin on his 86th birthday in Moscow.

\section{Honours}

Barenblatt held foreign memberships at the US National Academy of Sciences, the US National Academy of Engineering, the American Academy of Arts and Sciences and the Royal Society of London, as well as a long list of scientific societies in multiple countries. The long list of his honours and awards includes the G.I.Taylor Medal of the US Society of Engineering Science, the J.C. Maxwell Medal and Prize of the International Congress for Industrial and Applied Mathematics and the Timoshenko Medal of the American Society of Mechanical Engineers. ${ }^{1}$

\section{Writings}

Barenblatt was a superb and dedicated writer. His books include the following:

- Flow, Deformation and Fracture. Cambridge University Press, Cambridge, UK, 2014.

- Scaling. Cambridge University Press, Cambridge, UK, 2003.

- Dimensional Analysis. Gordon and Breach NY, USA, 1987.

- (With V.M. Entov and V.M. Ryzhik), The Motion of Fluids and Gases in Natural Rocks. (in Russian) Nedra, Moscow, 1984; (in English) Kluwer, 1990.

- (With Ya.B. Zel'dovich and G.M. Makhviladze), The Mathematical Theory of Combustion and Explosions. (in Russian) Nauka, Moscow, USSR, 1980; (in English) Pleanum Press, NY and London, 1985.

- (With A.P. Lisitzin) Hydrodynamics and Sedimentation. (in Russian) Nauka, Moscow, 1983.

- Scaling, Self-Similarity, and Intermediate Asymptotics. (with a foreword by Ya.B. Zel'dovich) (in Russian), Gidrometeoizdat, Leningrad, USSR, 1978, 1982; (in English) Plenum Press, NY, USA 1979; Cambridge University Press, Cambridge, UK, 1996.

- Dimensional Analysis and Self-Similar Solutions (in Russian), USSR Academy of Sciences, Moscow, 1975.

1 See full listing in http://math.lbl.gov/barenblatt/barenblatt_ paper_mono.html 
- (With V.M. Entov and V.M. Ryzhik) Theory of Unsteady Filtration of Fluids and Gases, (in Russian) Nedra, Moscow, 1972.

He was also co-editor of a number of books and author of numerous scientific publications dating from 1952 to his late years.

\section{Comments on his scientific work and legacy}

One of the major themes of Grigory Isaakovich's work over the years has been the development and application of scaling ideas to a variety of problems. Much of this work grew out of his seminal work with Ya.B. Zel'dovich on self-similarity as intermediate asymptotics. This was summarised in a remarkable review article (G.I. Barenblatt and Ya.B. Zel'dovich, "Self-similar solutions as intermediate asymptotics", Annual Review of Fluid Mechanics, 4 (1) (1972), pp. 285-312), which brought to a Western audience three under-appreciated ideas. Firstly, the conventional use of what is called "dimensional analysis" to simplify problems when one dimensionless group is negligible, makes an assumption about regularity that is frequently unjustified. When this assumption is removed, paradoxes can be resolved and new scaling laws emerge with non-rational exponents. In these problems, there is "scale-interference": the equation retains the memory of initial conditions, for example, even at asymptotically large time, in contrast to naive expectations. Secondly, these examples of so-called incomplete similarity can be dynamical attractors for a wide range of initial conditions and are associated with degenerate initial conditions that are generalised solutions (but not delta-functions). These attractors describe the dynamics for intermediate times between when initial transients have decayed and when the system reaches its final state, and hence are called intermediate asymptotics. Thirdly, these similarity solutions are, in fact, special cases of rather general Lie group symmetries. An important example is the analysis of travelling waves, where the front interpolates between a stable and an unstable fixed point. The speed can then be mapped by a simple change of variable into an exponent associated to a solution with incomplete similarity. This insight pertained especially to the famous waves analysed by Kolmogorov, Piskunov and Petrovsky in 1937. On the wall of his home office in Berkeley, Grigory Isaakovich had photos of the first and last authors of this work, along with several other individuals who meant a lot to him.

Barenblatt also speculated that there could be some connection between incomplete similarity and the problems of critical exponents at second-order phase transitions. The latter problem exercised the theoretical physicists around the world during the 1960s and 1970s, perhaps nowhere more so than in Moscow. This link was indeed later established directly by one of the article's authors (NG) and co-workers, showing inter alia how the elasto-plastic porous medium equation bearing Barenblatt's name could be solved by renormalisation group methods and providing analytic series expansion formulae for the exponents arising in its intermediate asymptotics. Grigory Isaakovich singled out these developments as a partial motivation for the updated revision of his classic book Similarity, Self-similarity and Intermediate Asymptotics, which proved to be highly influential in the West.

In Berkeley, he focused on the possibility that incomplete similarity would provide a more faithful description of the "intermediate" region in bounded shear flows, i.e. of the region not immediately adjoining the wall but not yet far from the wall, as well as a coherent theory of corrections to Kolmogorov's scaling in the inertial range of turbulence. The shear flow problem is of great practical importance - the scaling law for the intermediate layer provides a crucial input for a wide variety of computational models, for example, in the tracking of hurricanes and in the design of combustion engines. Grigory Isaakovich and his associate V.M. Prostokishin had deduced the coefficients in the scaling by processing experimental data and comparing the resulting laws to the data. This showed that the incomplete similarity solution fit the data to within experimental error in all cases, unlike the older, simpler scaling law (known as "the law of the wall"). Also, unlike in previous scaling laws, the parameters assumed to be constant remained constant in all cases, unlike the "constants" in the previous law, which varied by as much as $30 \%$ from one flow to the other. This left theoretical work to be done, showing that the resulting intermediate layer is asymptotically consistent with the rest of the flow and providing examples where the new scaling can be checked analytically. This was done during Grigory Isaakovich's stay in Berkeley in collaboration with AJC.

Kolmogorov's scaling of the intermediate ("inertial") scales of turbulence far from boundaries is the foundation of all theories of turbulence, yet experiments show that it is not exact and its derivation has been challenged many times, in particular by L.D. Landau. Kolmogorov himself eventually offered a "corrected" theory that has not been widely accepted. Barenblatt showed that incomplete similarity provides a self-consistent viscositydependent correction that converges to the original Kolmogorov solution in the limit of vanishing viscosity. This is a novel idea, which is well supported by the available experimental data.

A recurrent theme of his research was the use of similarity methods in wide classes of nonlinear heat equations known in the Russian literature as filtration equations. A simple nonlinear version of the heat equation that first comes to mind is the following equation: $\partial_{t} u=\Delta\left(u^{m}\right)$, with $m>1$, which is usually called the Porous Medium Equation (PME). It is relevant since it is nonlinear and, moreover, it is degenerate parabolic at the level $u=0$ so that the diffusion coefficient is just $m u^{m-1}$. Hence, it can serve as a model of restricted propagation that leads to free boundaries, sharp fronts with finite speed of propagation. It has been studied in $d$-dimensional Euclidean space, with interest in the cases $d=1,2,3$ for the applied scientist and with no dimension restriction for the mathematician. $\Delta$ represents the Laplace operator acting on 
the space variables. This equation appears in the description of different natural phenomena and its theory and properties depart strongly from the classical heat equation, $\partial_{t} u=\Delta u$, its most famous relative - hence the interest in its study, both for the pure mathematician and the applied scientist. There are a number of physical applications where this simple model appears in a natural way, mainly to describe processes involving fluid flow, heat transfer or diffusion. Maybe the best known of them is the description of the flow of an isentropic gas through a porous medium, modelled independently by the engineers L.S. Leibenzon in the USSR and M. Muskat in the USA around 1930. An earlier application is found in the study of groundwater infiltration by Boussisnesq in 1903. Another important application refers to heat radiation in plasmas, developed by Zel'dovich and co-workers around 1950. Indeed, this application was at the base of the rigorous mathematical development of the theory. Other applications have been proposed in mathematical biology, the spread of viscous fluids, boundary layer theory and other fields.

Serious progress in understanding this equation was reached around 1950 in Moscow by Zel'dovich, Kompaneets and Barenblatt, who found and analysed a solution representing heat release from a point source. This solution has an explicit formula in self-similar form $U(x, t)=t^{-\alpha} \mathrm{F}\left(|x| t^{-\beta}\right)$, with similarity exponents determined by the algebra of the equation and mass conservation. They found that the profile $F$ has the explicit form of an inverted parabola cut at the level $u=0$ so that two important properties are manifested: firstly, there exists a free boundary located at a distance $|x|=c t^{\beta}$ and secondly, the solutions are not differentiable enough to be classical. Since $\beta$ turns out to be less than $1 / 2$ for $m>1$, the propagation does not follow the Brownian scaling and the process can be considered as a relevant example of anomalous diffusion. Barenblatt not only did the rigorous analysis of these solutions (which now usually bear his name) in his 1952 paper but also found the selfsimilar source-type solution for the nonlinear heat equation with gradient diffusivity that we now call the evolution $p$-Laplacian equation (EPLE).

The PME equation has had enormous success with pure and applied mathematicians after Oleinik and collaborators supplied the first proof of existence, uniqueness and finite propagation of non-negative solutions of the PME in 1D in 1958. Gradually, the theory was developed with increasing generality in the last decades of the 20th century, much to Grigory Isaakovich's surprise and delight, and the results bear distinguished names: Kamin and Friedman supplied the first asymptotic proof; Bénilan, Brezis, Crandall, Evans and Pierre were busy with the construction of solutions with quite general data and the corresponding nonlinear semigroup theory; Angenent, Aronson, Caffarelli, DiBenedetto, Friedman, Sacks and others supplied the regularity theory for solutions and interfaces; and Peletier and collaborators supplied new self-similar solutions. Parallel progress occurred for the EPLE. Based on all this knowledge, DiBenedetto wrote a book on Degen-

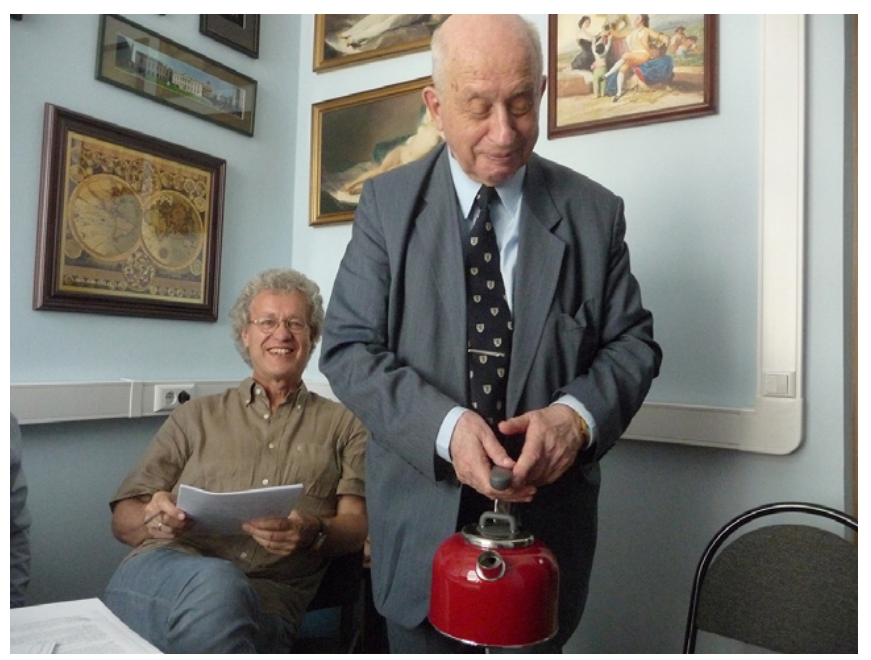

In his office in the Shirshov Institute of Oceanology, Moscow 2013.

erate Diffusions (1993) and one of the article's authors (JLV) wrote a book describing the state of the art for the PME (Oxford Univ. Press, 2007). This goes to show the enormous influence of the ideas originally coming from Zel'dovich and given mathematical treatment by Barenblatt.

In the current century, Barenblatt's ideas have had an important impact on the theory of nonlinear PDEs in various directions. One was the growth of entropy methods, coming from statistical mechanics and used to prove the convergence of general solutions to stable profiles. The techniques of scaling, so dear to Grigory Isaakovich, made possible the use of entropy methods on nonlinear heat equations of the previous types to establish intermediate asymptotics to Barenblatt solutions with rates (we refer to work by Markowich, Toscani, Carrillo, del Pino, Dolbeault, Bonforte, Grillo, etc.). A second direction is the study of nonlocal diffusion, based mainly on the use of fractional Laplacian operators. The name "Barenblatt solutions" appears in a number of models of nonlinear, nonlocal models as self-similar profiles evolving from a point-mass initial datum, and asymptotic convergence proofs have been found for more general data. One of the article's authors (JLV) has been intensely involved in such directions and has been witness to the sense of wonder that this ongoing "Barenblatt activity" always produced in Grigory Isaakovich. A third direction derives from the mathematical treatment of heat and mass transfer in stably stratified turbulent shear flow, where the physically relevant regularisation of an ill-posed diffusion equation is still so degenerate that solutions may become discontinuous. This has led to a systematic study of discontinuous transient solutions of nonlinear PDEs, mainly by a PDE group in Rome that was strongly influenced by Barenblatt's continuous encouragement to develop PDE theories motivated by his models of turbulence, flow in porous media, thin liquid films and damage accumulation. In this sense, Grigory Isaakovich's enthusiasm had no limits: he asked such PDE questions even during his last years at the Oceanology Laboratory in Moscow, 
where Joost Hulshof, Grigory Isaakovich's collaborator and friend from Amsterdam, and one of the article's authors (MB) had the privilege to visit him more than once to discuss turbulent shear flows with suspended particles.

The writers of this article think that Grisha's life adventure will endure as a brilliant tale of the fruitful encounter of two worlds, a rare event that we were fortunate enough to witness and that was, in large part, due to his immense curiosity. Grisha held clear ideas about the need for a strong engineering-physics-mathematics interaction that includes pure mathematics, and also about the need for a strong connection between science and culture and for a better understanding between cultures. He will always remain in our memory as an example and a scientific hero.

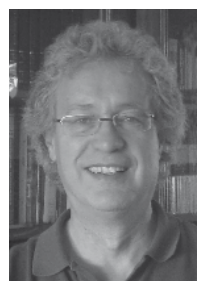

Michiel Bertsch has been a professor of mathematical analysis at the University of Rome Tor Vergata since 1990. Between 1997 and 2014, he headed the Istituto per le Applicazioni del Calcolo "Mauro Picone"(CNR) in Rome. His research area is nonlinear PDEs and their applications.

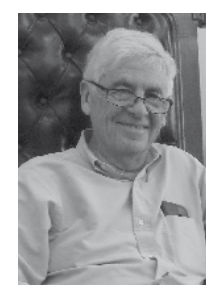

Alexandre J. Chorin is an emeritus professor of mathematics at the University of California at Berkeley, where he has worked since 1971, and a senior research scientist at the Lawrence Berkeley National Laboratory. He is a member of the US National Academy of Sciences. His research areas are computational fluid dynamics and computational statistical mechanics.

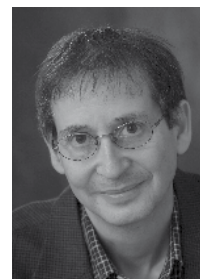

Nigel Goldenfeld has been a professor of theoretical physics at the University of Illinois at Urbana-Champaign since 1985. He is a member of the US National Academy of Sciences. His research areas include statistical mechanics and renormalisation group theory, condensed matter theory and the physics of living systems.

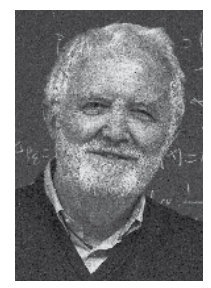

Juan Luis Vázquez has been a professor of applied mathematics at the University Autónoma de Madrid from 1986 to 2016 and is now an emeritus professor and member of the Spanish Royal Academy of Sciences. $H$ is research areas are nonlinear PDEs, free boundaries and asymptotic methods. 